\title{
-NOTES-
}

\section{SOME APPLICATIONS OF THE REPEATED INTEGRALS OF THE ERROR FUNCTION*}

\author{
By J. C. JAEGER (University of Tasmania)
}

1. Introductory. The repeated integrals of the error function

where

$$
\mathrm{i}^{n} \operatorname{erfc} x=\int_{x}^{\infty} \mathrm{i}^{n-1} \operatorname{erfc} \xi d \xi, \quad n=1,2, \cdots
$$

$$
\mathrm{i}^{0} \operatorname{erfc} x=\operatorname{erfc} x=\frac{2}{\sqrt{\pi}} \int_{x}^{\infty} e^{-\xi^{2}} d \xi,
$$

have been studied by Hartree, ${ }^{1}$ who tabulates them for $n=1$ and $n=2$, and shows that they satisfy the recurrence relation

$$
2 n \mathrm{i}^{n} \text { erfc } x=\mathrm{i}^{n-2} \operatorname{erfc} x-2 x \mathrm{i}^{n-1} \operatorname{erfc} x .
$$

He also shows that

$$
L\left\{(4 t)^{n / 2 \mathrm{i}^{n}} \operatorname{erfc}\left(\frac{1}{2} a t^{-1 / 2}\right)\right\}=s^{-1-n / 2} e^{-a \sqrt{s}},
$$

where $n=0,1,2, \cdots, a \geqq 0$, and $L\{v\}$ is written for the Laplace transform of a function $v(t)$ of $t$, that is

$$
L\{v\}=\int_{0}^{\infty} e^{-s t} v(t) d t
$$

The functions (1) arise naturally in the theory of conduction of heat in the semiinfinite solid (or the sphere or slab) with prescribed surface temperature or flow of heat, since the Laplace transforms of the solutions of many such problems involve the functions on the right hand side of (4).

The objects of this note are, firstly, to indicate an extension of (4) which applies in the same way to problems with heat transfer at the surface, and, secondly, to give solutions in terms of the functions (1) of a number of problems of practical interest which involve heat generation in the solid.

2. Problems involving heat transfer at a surface at a rate proportional to its temperature difference from its surroundings. The required extension of (4) is that, if $n$ is a positive integer, and $\alpha, h$, and $x$ are positive,

$$
L\left\{\alpha(-h)^{-n}\left[e^{2 H X+H^{2}} \operatorname{erfc}(H+X)-\sum_{r=0}^{n-1}(-2 H)^{r^{r} r} \operatorname{erfc} X\right]\right\}=\frac{e^{-q x}}{q^{n+1}(q+h)},
$$

where

$$
X=x / 2 \sqrt{\alpha t}, \quad H=h \sqrt{\alpha t}, \quad q=\sqrt{s / \alpha} .
$$

* Received Sept. 19, 1945.

' D. R. Hartree, Some properties and applications of the repeated integrals of the error function, Proc. Manchester Lit. and Phil. Soc. 80, 85 (1935). 
To derive this result we notice that

$$
\frac{e^{-q x}}{q^{n+1}(q+h)}=\frac{(-)^{n} e^{-q x}}{h^{n} q(q+h)}+\frac{e^{-q x}}{h^{n} q^{2}} \sum_{r=0}^{n-1}(-)^{n-r-1}\left(\frac{h}{q}\right)^{r} \text {. }
$$

In the terms of the series we use (4); the result for the first term of the right-hand side of (8) is given in most tables of Laplace transforms; and (6) follows immediately.

Typical examples in which (6) arises are the following:

(i) The semi-infinite solid $x>0$. Zero initial temperature. The solid heated at $x=0$ for $t>0$ by heat transfer from a medium at at ${ }^{n / 2}, n=0,1, \cdots$.

The temperature $v$ in the solid has to satisfy

$$
\frac{\partial^{2} v}{\partial x^{2}}-\frac{1}{\alpha} \frac{\partial v}{\partial t}=0, \quad x>0, \quad t>0,
$$

with the boundary condition

$$
\frac{\partial v}{\partial x}=-h\left(a t^{n / 2}-v\right), \quad x=0, \quad t>0 .
$$

Also $v$ has to be bounded as $x \rightarrow \infty$. The Laplace transform of the solution is found to be

$$
L\{v\}=\frac{h a \Gamma(1+n / 2) e^{-q x}}{s^{1+n / 2}(q+h)},
$$

using the notation (7). Therefore, from (6)

$$
v=\frac{(-)^{n+1} a \Gamma(1+n / 2)}{h^{n} \alpha^{n / 2}}\left\{e^{2 H X+H^{2}} \operatorname{erfc}(H+X)-\sum_{r=0}^{n}(-2 H)^{r i r} \operatorname{erfc} X\right\} .
$$

If heat transfer takes place from a medium whose temperature is

$$
\sum_{n=0}^{N} a_{n} t^{n / 2}
$$

the solution follows at once. For the problem of the semi-infinite solid whose surface temperature is given by (10) the result is obtained in the same way by using (4). An empirical relation of the type (10) is often useful for representing observed surface temperatures, the term in $t^{1 / 2}$ being particularly valuable since it corresponds to constant flux of heat; for example the fall in temperature of the Earth's surface after sunset on a cloudless evening is approximately proportional to $t^{1 / 2}$ and may be represented very well by two or three terms of (10).

(ii) The semi-infinite solid $x>0$, of conductivity $K$ and diffusivity $\alpha$. At $x=0$ the solid is in contact with mass $M$ per unit area of well stirred fluid of specific heat $c^{\prime}$, whose temperature is equal to the surface temperature of the solid, i.e. to $\lim _{x \rightarrow+0} v$. The initial temperatures of the solid and fluid are zero. Heat is supplied to the fluid at constant rate $Q$ per unit mass per unit time for $t>0$.

Here (9) has to be solved with boundary condition at $x=0$

$$
M c^{\prime} \frac{\partial v}{\partial t}-K \frac{\partial v}{\partial x}=Q M, \quad x=0, \quad t>0 .
$$


The Laplace transform of the solution is

$$
L\{v\}=\frac{Q e^{-q x}}{\alpha^{2} c^{\prime} q^{3}(q+h)},
$$

where now $h=K / M c^{\prime} \alpha$. Thus from (6) we have

$$
v=\frac{Q}{h^{2} \alpha c^{\prime}}\left\{e^{2 H X+X^{2}} \operatorname{erfc}(H+X)-\operatorname{erfc} X+2 H \mathrm{i}^{1} \operatorname{erfc} X\right\} .
$$

(iii) The semi-infinite solid $x>0$. Zero initial temperature. Heat is produced for $t>0$ in the solid at the rate $Q t^{n / 2}, n=-1,0,1, \cdots$, per unit time per unit volume. There is heat transfer at $x=0$ into a medium at zero temperature.

Here we have to solve the differential equation

$$
\frac{\partial^{2} v}{\partial x^{2}}-\frac{1}{\alpha} \frac{\partial v}{\partial t}=-\frac{Q}{K} t^{n / 2}, \quad x>0, t>0,
$$

with boundary condition

Here

$$
\frac{\partial v}{\partial x}-h v=0, \quad x=0, \quad t>0 .
$$

$$
L\{v\}=\frac{Q \alpha \Gamma(1+n / 2)}{K}\left\{\frac{1}{s^{2+n / 2}}-\frac{h e^{-q x}}{\alpha^{2+n / 2} q^{n+4}(q+h)}\right\},
$$

and

$v=\frac{Q \alpha t^{1+n / 2}}{K(1+n / 2)}+\frac{Q \Gamma(1+n / 2)}{K \alpha^{n / 2}(-h)^{n+2}}\left[e^{2 H X+H^{2}} \operatorname{erfc}(H+X)-\sum_{r=0}^{n+2}(-2 H)^{r} \mathrm{i}^{r} \operatorname{erfc} X\right]$.

3. Cases of generation of heat in a solid. The solutions of a number of problems of practical importance in which heat is generated for $t>0$ in a solid at the rate

$$
\sum_{n=1}^{N} a_{n} t^{n / 2}
$$

per unit time per unit volume can be expressed in terms of the functions (1). An expression of type (11) may be useful for representing an experimentally observed rate of generation of heat; the term in $t^{-1 / 2}$ is of value when the initial rate of heat production is high, as in the hydrating of cement.2

A problem involving (11) and radiation at the surface has already been given in $\$ 2(\mathrm{iii})$, here we give the solutions of some cases in which the surface temperature is zero.

(iv) The region $x>0 . x=0$ kept at zero for $t>0$. Heat production at the rate $t^{n / 2}$ in $0<x<a$, and zero in $x>a$. Zero initial temperature.

2 In this case two or three terms of (11) give quite a good representation of the observed heat of hydration over the first few days. An expression in terms of negative exponentials is more usual, but does not lead to solutions in terms of tabulated functions for the problems given here, except (vi), and in that case it does not give a solution which is useful for small values of the time. 
This is the fundamental practical problem of temperatures in hydrating concrete: a slab of concrete is poured on the surface of the semi-infinite solid, we assume here that the thermal constants of the concrete and the solid are the same. The solution is $v=\frac{\alpha t^{1+n / 2}}{K(1+n / 2)}\left\{1-\Gamma(2+n / 2) 2^{n+1}\left[\mathrm{i}^{n+2} \operatorname{erfc} \frac{a-x}{2(\alpha t)^{1 / 2}}-\mathrm{i}^{n+2} \operatorname{erfc} \frac{a+x}{2(\alpha t)^{1 / 2}}\right.\right.$

if $0<x<a$, and for $x>a$ it is

$$
\left.\left.+2 \mathrm{i}^{n+2} \operatorname{erfc} \frac{x}{2(\alpha t)^{1 / 2}}\right]\right\}
$$

$$
\begin{aligned}
v=\frac{\alpha \Gamma(1+n / 2)(4 t)^{1+n / 2}}{2 K}\left\{\mathrm{i}^{n+2} \operatorname{erfc} \frac{x-a}{2(\alpha t)^{1 / 2}}+\mathrm{i}^{n+2} \operatorname{erfc} \frac{x+a}{2(\alpha t)^{1 / 2}}\right. & \left.-2 \mathrm{i}^{n+2} \operatorname{erfc} \frac{x}{2(\alpha t)^{1 / 2}}\right\} .
\end{aligned}
$$

(v) The problem ${ }^{3}$ of (iv) except that heat is produced only in the region $a<x<b$.

The temperature gradient at the surface is

$$
\frac{\alpha^{1 / 2}}{K} \Gamma(1+n / 2)(4 t)^{(1+n) / 2}\left[\mathrm{i}^{1+n} \operatorname{erfc} \frac{a}{2(\alpha t)^{1 / 2}}-\mathrm{i}^{1+n} \operatorname{erfc} \frac{b}{2(\alpha t)^{1 / 2}}\right] .
$$

(vi) The slab $0<x<l$. The surfaces $x=0$ and $x=l$ kept at zero temperature. Heat generation at the rate $t^{n / 2}$. Zero initial temperature.

$$
\begin{aligned}
v=\frac{\alpha t^{1+n / 2}}{K(1+n / 2)}\left\{1-\Gamma(2+n / 2) 2^{n+2} \sum_{m=0}^{\infty}(-)^{m}\left[\mathrm{i}^{n+2} \operatorname{erfc}\right.\right. & \frac{m l+x}{2(\alpha t)^{1 / 2}} \\
& \left.\left.+\mathrm{i}^{n+2} \operatorname{erfc} \frac{(m+1) l-x}{2(\alpha t)^{1 / 2}}\right]\right\} .
\end{aligned}
$$

(vii) The infinite region $r \geqq 0$. Zero initial temperature. Heat production at the rate $t^{n / 2}$ in the sphere $0 \leqq r<a$, zero elsewhere. ${ }^{4}$

$$
\begin{aligned}
v= & \frac{\alpha t^{1+n / 2}}{K(1+n / 2)}-\frac{\alpha a \Gamma(1+n / 2)(4 t)^{1+n / 2}}{2 K r}\left[\mathrm{i}^{n+2} \operatorname{erfc} \frac{a-r}{2(\alpha t)^{1 / 2}}-\mathrm{i}^{n+2} \operatorname{erfc} \frac{a+r}{2(\alpha t)^{1 / 2}}\right] \\
& -\frac{\alpha^{3 / 2} \Gamma(1+n / 2)(4 t)^{(n+3) / 2}}{2 K r}\left[\mathrm{i}^{n+3} \operatorname{erfc} \frac{a-r}{2(\alpha t)^{1 / 2}}-\mathrm{i}^{n+3} \operatorname{erfc} \frac{a+r}{2(\alpha t)^{1 / 2}}\right], 0 \leqq r<a . \\
v= & \frac{\alpha a \Gamma(1+n / 2)(4 t)^{1+n / 2}}{2 K r}\left[\mathrm{i}^{n+2} \operatorname{erfc} \frac{a+r}{2(\alpha t)^{1 / 2}}+\mathrm{i}^{n+2} \operatorname{erfc} \frac{r-a}{2(\alpha t)^{1 / 2}}\right] \quad r>a . \\
& +\frac{\alpha^{3 / 2} \Gamma(1+n / 2)(4 t)^{(n+3) / 2}}{2 K r}\left[\mathrm{i}^{n+3} \operatorname{erfc} \frac{a+r}{2(\alpha t)^{1 / 2}}-\mathrm{i}^{n+3} \operatorname{erfc} \frac{r-a}{2(\alpha t)^{1 / 2}}\right], \quad r>a
\end{aligned}
$$

${ }^{3}$ Van Ostrand, On the flow of heat from a lock stratum in which heat is being genesated, J. Wash. Acad. Sci. 22, 529 (1932), considers the case of a thin layer.

- This problem is of interest in connection with development of heat in wheat stacks. 